\title{
MYOCARDIAL TUBERCULOSIS WITH PAROXYSMAL VENTRICULAR TACHYCARDIA
}

\author{
BY \\ ROBERT SCHNITZER \\ From the Essex County Hospital, Wanstead \\ - Received April 4, 1947
}

Tuberculous myocarditis is extremely rare, and even in tuberculous pericarditis it is uncommon. The reason for this is difficult to explain. Since the lungs are a common site of tuberculous infection, one might expect myocardial infection to occur much more frequently. It has been suggested that the constant movement of the musculature is not conducive to the lodgement of tubercle bacilli or to the development of tubercles, and that the lactic acid produced by cardiac muscular activity offers some protection against Koch's bacilli (Raviart, 1906). The following unusual case is reported.

\section{CASE RECORDS}

A Petty Officer in the French Navy, aged 32, was admitted to hospital in August 1945. He looked extremely ill and slightly cyanosed and complained of severe breathlessness and palpitation.

History. He had always enjoyed good health. In 1940, during the evacuation from Dunkirk, he was wounded in the left forearm and subsequently the whole limb had to be amputated and he was fitted with an artificial arm. Soon afterwards he began to complain of tiredness and shortness of breath on the slightest exertion and this was followed by attacks of palpitation. These attacks started and stopped abruptly, and were accompanied by severe dyspnœa, an aching sensation in the amputation stump, and occasionally by slight hæmoptysis. He had lost $12 \mathrm{lb}$. in weight within five months and was sent to a sanatorium for further investigation. $\mathrm{X}$-rays of his chest suggested some infiltration in both lungs but were at first not quite typical of tuberculosis, and repeated sputum examination, including cultures, failed to reveal acid-fast bacilli. A bronchogram showed no abnormality. After his discharge from the sanatorium he experienced a severe attack of paroxysmal tachycardia in which he was admitted to hospital twelve hours after the onset.

Examination. A youthful looking man, ashen-grey and cyanosed, dyspnœic, and sweating profusely. Temperature $99 \cdot 4^{\circ} \mathrm{F}$., pulse very rapid and regular; respiration 36 . Neck veins distended; apex beat in sixth inter space $3.5 \mathrm{~cm}$. outside mid-clavicular line; no murmurs. Blood pressure $130 / 80 \mathrm{~mm}$. No clubbing of fingers. Trachea central. A few bronchitic sounds scattered over both lungs, especially over the right mid-zone. Liver not enlarged; spleen not palpable; no œdema.

Progress. Quinidine, intramuscularly and orally, and carotid sinus pressure failed to stop the paroxysm and acute pulmonary œema developed. Morphine had only a limited effect. A cardiogram during this attack showed paroxysmal ventricular tachycardia (P.V.T.) with a ventricular rate of 280-300 a minute, similar but not identical with the paroxysm shown later. X-ray of the chest suggested infiltration in the right lung, and to a lesser degree in the left, and some enlargement of the paratracheal gland. Intravenous injection of $0.5 \cdot \mathrm{mg}$. 


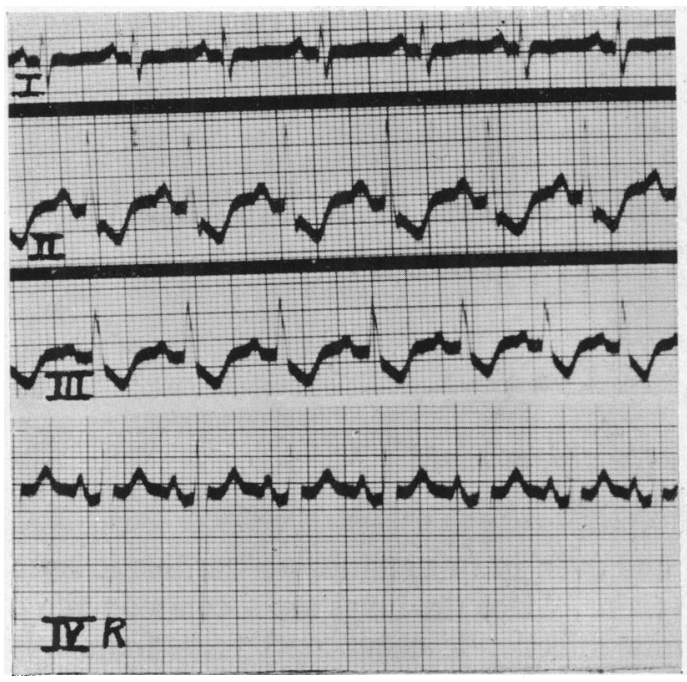

FIG. 1.

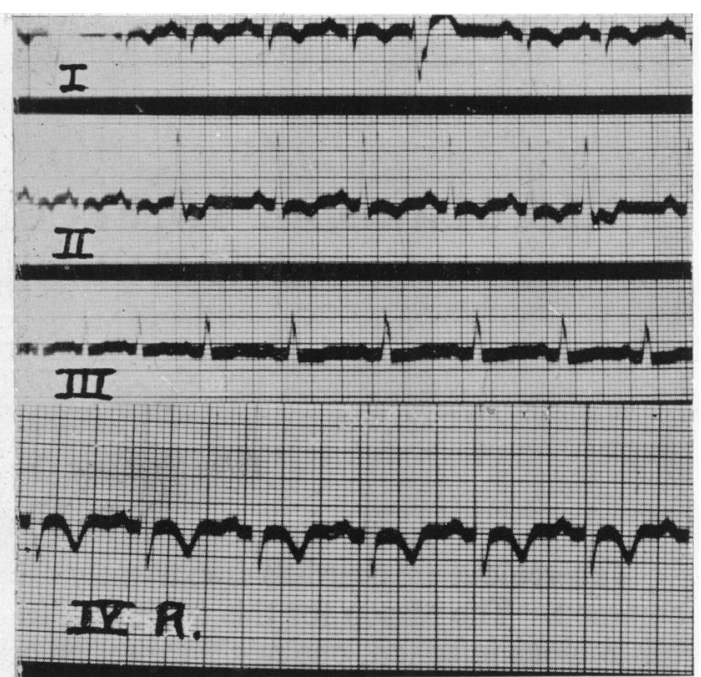

FIG. 2.

Fig. 1.-Electrocardiogram after a paroxysm that lasted 36 hours. Tachycardia ceased after injection of $0.5 \mathrm{mg}$. digoxin intravenously. Changes suggest myocardial infarction of T III type, or possible effect of recent paroxysm, or of digitalis.

FIG. 2.-Two weeks later. Changes suggest myocardial infarction of $\mathrm{T} I$ type.

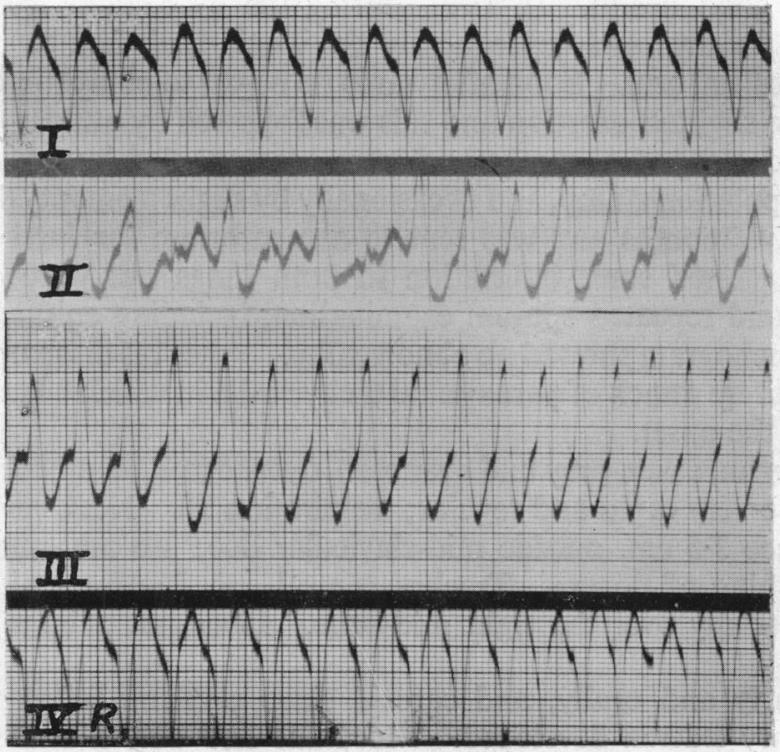

Fig. 3.

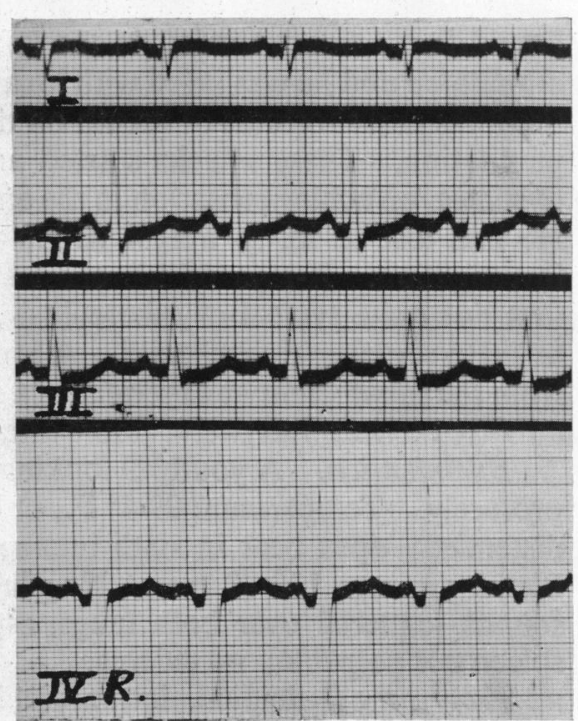

Fig. 4.

Fig. 3.-Paroxysm of ventricular tachycardia that lasted 5 hours.

FIG. 4.-Ten days before death. Changes seen in the previous tracings have almost completely subsided. 
digoxin reduced the apex rate to 120 , and within one hour a further drop to 108 was observed. Digitalis was continued by mouth in small doses and only a few short attacks of P.V.T. occurred, but after four days it had to be stopped because of frequent extrasystoles. Cardiograms between the attacks showed transient $T$ wave changes which might have been attributed to pulmonary infarction, to posterior cardiac infarction, to the effect of the paroxysmal tachycardia, or to digitalis (Fig. 1). Later, however, inversion of the T I suggested anterior cardiac infarction (Fig. 2). One night he complained of weakness and a sinking feeling, and tachycardia recurred and persisted until death early the next morning. A record of one of his paroxysms of ventricular tachycardia is shown in Fig. 3. A cardiogram taken only ten days before his death showed none of the changes seen in previous tracings (Fig. 4).

Investigations. On admission, white blood cells, 27,700 , with 89 per cent polymorphs; 10 days later, 5600 , with 71 per cent polymorphs. Blood sedimentation rate on admission, $25 \mathrm{~mm}$. in one hour (Westergren); 3 weeks later $10 \mathrm{~mm}$. in one hour. Blood urea $35 \mathrm{mg}$. per $100 \mathrm{ml}$. Wassermann and Kahn negative. Urine, slight traces of albumin with many pus cells; culture sterile. Sputum, tubercle bacilli not found; culture for tubercle bacilli negative.

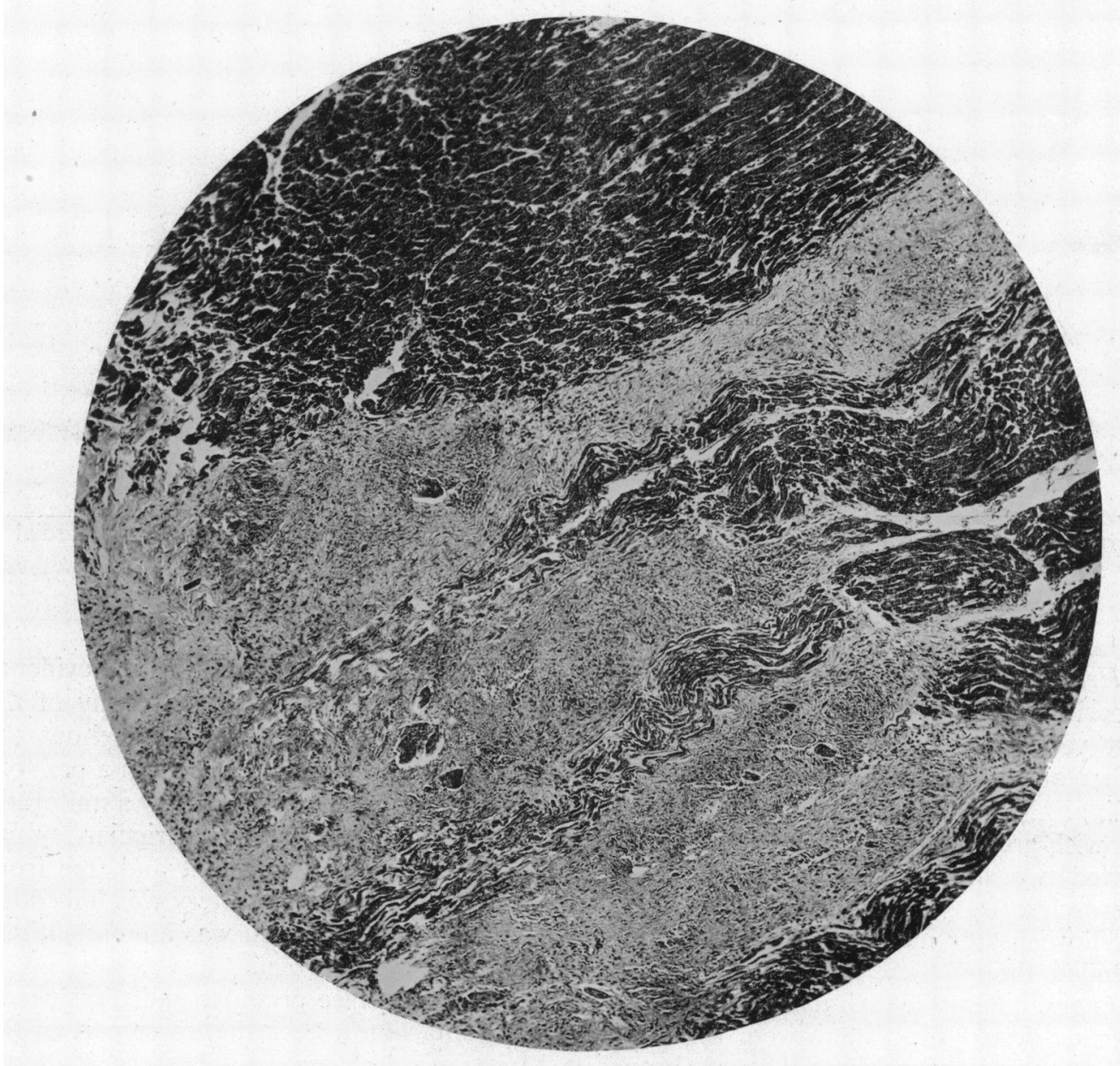

Fig. 5.-Section showing giant celled nodules in myocardium. Magnification: $\times 52$. 


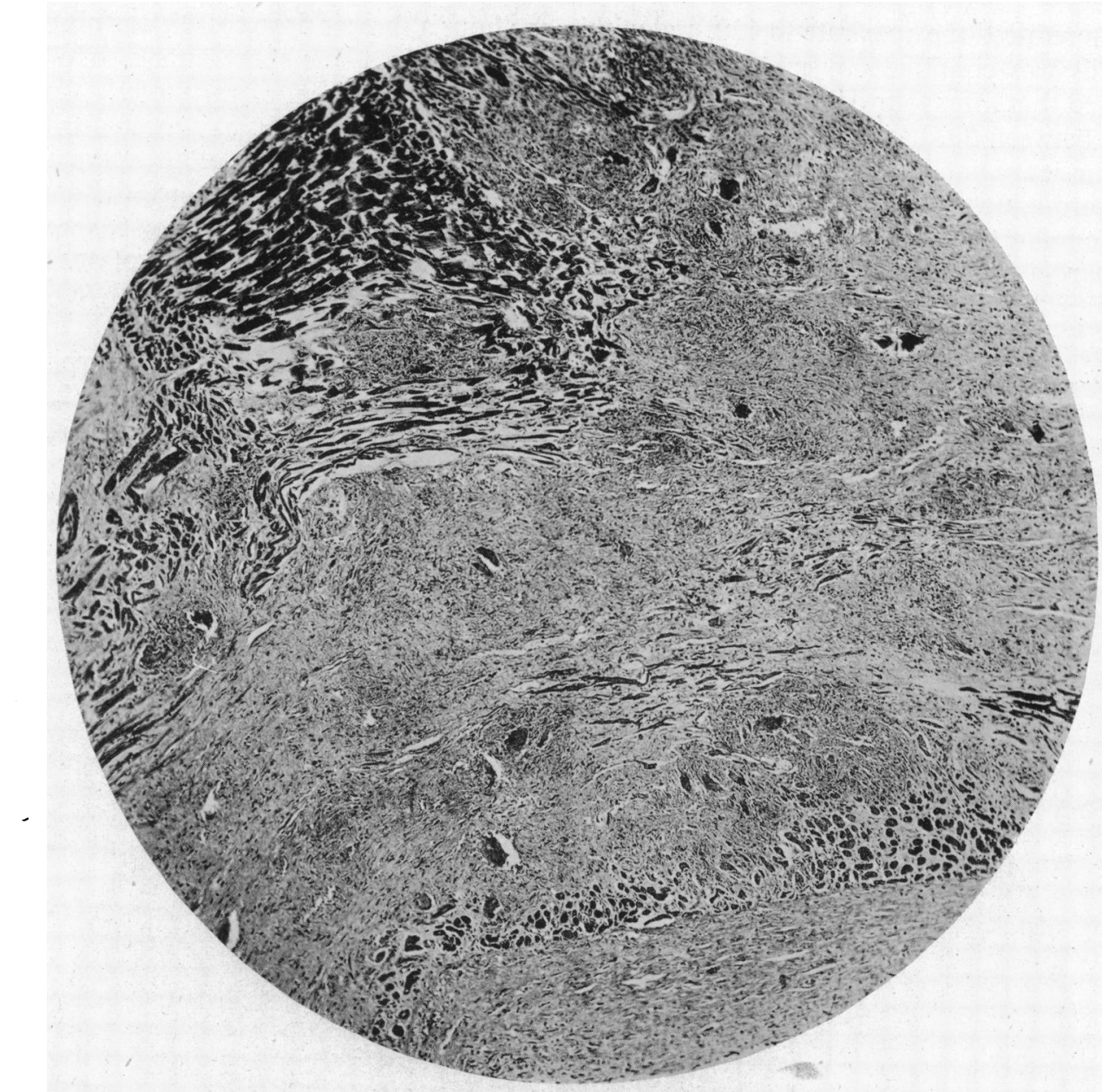

FIG. 6.-Section showing confluent caseous tuberculosis, and miliary foci between the lower myocardial fibres. Magnification: $\times 52$.

\section{POST-MORTEM EXAMINATION}

Heart. There was a small amount of straw-coloured pericardial effusion, but no evidence of pericarditis. There was some enlargement of the heart with slight hypertrophy of the right ventricle. There were multiple greyish and yellowish-grey patches throughout the surface of both ventricles; some had a fibrotic appearance and varied in size from a pea to a shilling. More than half of the anterior wall of the left ventricle was replaced by a uniformly yellowish-grey material which extended upward to the aorta, and the interventricular septum showed two similar smaller lesions. The right coronary artery was patent and free from atherosclerosis, but the anterior descending branch of the left coronary artery was imbedded in yellowish-grey material throughout most of its length and its lumen was almost entirely occluded, though there was no thrombosis. All the valves were normal.

Lungs. Paratracheal gland enlarged and firm, the cut surface granular and translucent, its tissue destroyed. There were white patches extending upwards to the lower part of the trachea and downwards into the main bronchi. Multiple greyish-green patches were scattered throughout both lungs, especially in the upper zones. No cavities were seen. 
The spleen was enlarged and soft, with multiple irregular greyish patches on the surface and in the parenchyma. The liver was not enlarged, but showed similar lesions and a few dark hæmorrhagic areas. Kidneys and adrenals were normal.

Histological report. Sections of the lung show numerous miliary and confluent tubercles with central caseation in some. Fibrosis is present within and around some isolated tubercles and is marked in the confluent areas, some of which are reduced almost to hyaline scars. Tuberculous arteritis is present in one small branch of the pulmonary artery. The remainder of the lung parenchyma shows œdema and congestion, and alveoli are occupied by an eosinophil coagulum containing free macrophages. A bronchial gland consists of an acellular mass of collagen, enclosing patches of amorphous débris and cholesterol crystals and surrounded by a capsule of fibrotic granulation tissue containing miliary tubercles.

Blocks from the left ventricle and interventricular septum of the heart show similar miliary and confluent miliary tubercles in the myocardium with fibrosis within and around the tubercles. In the left ventricle, fibrous granulation tissue containing tubercles replaces the outer part of the myocardium for a depth of at least $1 \mathrm{~cm}$. except for a few included groups of muscle fibres (Fig. 5 and 6). Inflammatory infiltration is slight in this confluent area, and consists of macrophages, lymphocytes, plasma cells, and a few neutrophil leucocytes.

The dark areas in the liver proved to be cavernous hæmangiomata and in one section measured up to $1.5 \mathrm{~cm}$. in diameter. The remainder of the liver in sections shows albuminous degeneration of the parenchymal cells and scattered miliary tubercles, one having a caseous centre. The spleen shows miliary and confluent miliary tubercles involving Malpighian bodies. The kidneys show albuminous degeneration of the tubular epithelium.

The tubercles in the lung, liver, spleen, and myocardium are composed of collagen, epithelioid and multinuclear giant histiocytes, fibroblasts, and a few lymphocytes. In many tubercles, most or all of the cells are necrosed, and in some the necrosed cells form a homogeneous caseous mass. Caseation affects only a part, usually central, of a tubercle. The giant cells are variable in appearance; some are typical Langhans's cells, but others are more of the foreign body type in that the nuclei, of which there are as many as 40 , are scattered and chiefly central. In all sites some giant cells contain eosinophil, crystalloid, star-like, radial cytoplasmic inclusions similar to, but probably not so well developed as, those in the StengelWolbach disease. Acid-fast bacilli could not be found in spite of prolonged search. The presence of caseation in many of the tubercles indicates almost certainly that the condition is tuberculous. The absence of tubercle bacilli might be expected in a tuberculous inflammation of such low grade intensity as was evident in this case.

\section{Discussion}

In the case described, the diagnosis of myocardial tuberculosis is established beyond doubt by the histological findings, despite the absence of tubercle bacilli from the sputum and from tissue sections. The route of infection is difficult to explain in the absence of pericarditis, though the lesions in the paratracheal gland, the bronchi and lungs, suggest a primary lymphatic spread. The tubercle bacilli may have gained access to the venous blood stream via the lymphatic duct, then infected the lungs and eventually entered the general circulation via the pulmonary capillaries, whence the myocardium, liver, and spleen became involved.

A case of myocardial tuberculosis was reported by Townsend (1832) who described "a tumour-like auricular growth." In Anders's (1902) extensive review of the subject, the total of reported cases was brought up to 72. From the time of Bollinger's report in 1890 and Raviart's study in 1906, 101 cases had been observed over a period of 16 years, while only 79 cases had been recognized previously. Norris (1904), in a study of 1764 hearts of tuberculous subjects, observed only 6 cases.

Tuberculosis of the myocardium is almost invariably secondary to a tuberculous focus 
elsewhere. In the pathogenesis of this disease, three possible routes of infection have been considered, $(a)$ by the blood stream, $(b)$ by retrograde lymph extension, and $(c)$ by contiguous tissues. Myocardial involvement is usually secondary to tuberculous pericarditis. Caseous tuberculous mediastinal lymph nodes, particularly the bronchial or paratracheal group, may be the source of infection of the pericardium and heart. Infection may occur by means of direct contact or through the lymphatics; this latter would necessitate a reversal of the lymph flow, since the drainage of the pericardium has been shown to be upwards into the bronchial lymph nodes (Recklinghausen, 1885). Another possible mode of spread is by direct extension from the pleura.

Three types of myocardial tuberculosis are usually recognized, the nodular, the miliary, and the diffuse infiltrating form. Raviart described a fourth type which he called "chronic interstitial tuberculous myocarditis"; this included the so-called "sclerosis of the myocardium." The existence of this last form has been disputed and it is generally held that fibrous myocardial lesions which may be found in the hearts of those dying from tuberculosis are not necessarily of tuberculous origin. Some such cases, in which tubercles or tubercle bacilli have been found in sections, probably belong to the diffuse infiltrative form of myocardial tuberculosis. The nodular variety is the commonest, and nodules varying from the size of a pea to that of an egg have been reported. Next in frequency is the miliary variety and rarest is the diffuse infiltrative form. According to Moenckeberg (1924), the last mentioned is a " specific diffuse productive tuberculous myocarditis " most frequently a sequel to pericarditis. Here the myocardium appears to be taken up by a uniformly grey or yellowgrey firm material, which may almost entirely replace the muscle in the areas involved. Most authors report that Ziehl-Neelsen staining has failed to reveal tubercle bacilli in spite of typical histological changes. The infiltrating type most often involves the auricles and may cause auricular extrasystoles and rarely auricular fibrillation (Sweeney, 1940). The ventricles are sometimes involved, and occasionally extensive destruction of the myocardium and conducting tissues has been observed. Caseous infiltration may destroy half or more of the thickness of the ventricular wall. Coronary occlusion is seldom observed in cases of tuberculosis, and tuberculous arteritis has been observed very rarely in the coronary arteries where it is usually confined to the smaller branches. In one reported case, however, a comparatively large coronary branch was involved, and, in the present case, almost the whole length of the left descending coronary artery was surrounded and partly occluded by tuberculous infiltration. The initial leucocytosis of 27,700, which disappeared within 10 days, may have been due to the ventricular tachycardia rather than to coronary occlusion; for a leucocytosis of 20,000 to 30,000 which subsides rapidly after termination of the paroxysm may be a feature of paroxysmal tachycardia (Levine and Golden, 1922).

\section{SUMMARY}

A case of myocardial tuberculosis complicated by paroxysmal ventricular tachycardia is reported. X rays showed pulmonary infiltration, but sputum tests for tubercle bacilli were negative.

Necropsy showed miliary tuberculosis with extensive involvement of the myocardium, including the interventricular septum, without pericarditis. The tuberculous nature of the lesions in the heart and other organs was established by the histological findings, which are described.

The publications relating to tuberculous myocarditis are briefly reviewed and the pathology is discussed.

I wish to thank Dr. O'Brien and Dr. J. Gilmour for their detailed histological report, and Dr. M. Brown and Dr. D. Gutman for their assistance at the necropsy. I am also indebted to Dr. John Parkinson and Dr. J. W. Linnell for their interest and help, and to Dr. P. J. W. Mills, Medical Superintendent, Lister Emergency Hospital. Hitchin, for permission to publish this case. 


\section{REFERENCES}

Anders, J. M. (1902). J. Amer. med. Ass., 39, 1081.

Bollinger, O. (1890). Münch. med. Wschr., 37, 567.

Levine, S. A., and Golden, R. (1922). Arch. intern. Med., 29, 836.

Moenckeberg, J. G. (1924). Handbuch der Speciellen. Path. Anat. und. Histol. Henke-Lubarsch, Berlin, Julius Springer.

Norris, G. W. (1904). Amer. J. med. Sci., 128, 649.

Raviart, G. (1906). Arch. Méd. expér., 18, 141.

Recklinghausen, R. (1885). Virchows Arch., 100, 503.

Sweeney, J. A. (1940). Amer. Heart. J., 20, 345.

Townsend, R. (1832). $\quad$ Dublin J. med. Sci., 1, 176. 